\title{
Multifocal epithelial hyperplasia (Heck disease) in a 7-year-old boy
}

\author{
Alexander Kreuter MD, Steffi Silling PhD
}

- Cite as: CMAJ 2018 December 17;190:E1484. doi: 10.1503/cmaj.180882

A 7-year-old boy from Angola presented at the dermatology department with a 2-year history of slowly enlarging, asymptomatic oral papules. The patient was otherwise healthy, and testing for immunodeficiency, including HIV, was negative. There were no similar lesions in other family members. Clinical examination showed numerous flat, white, coalescing papules and plaques on the lower labial mucosa (Figure 1). Otherwise, extraoral examination did not show any abnormalities. Histopathologic analysis from a representative lesion showed parakeratosis, acanthosis, marked epithelial hyperplasia and koilocytosis. The diagnosis of multifocal epithelial hyperplasia (or Heck disease) was supported by the presence of human papillomavirus (HPV) type 13 on polymerase chain reaction analysis.

This uncommon, benign disease of the oral mucosa is induced by an infection with HPV type 13 or HPV type $32 .{ }^{1}$ It predominantly affects children and young adults, with a female predilection. Multifocal epithelial hyperplasia occurs in certain geographical regions (e.g., North, Central and South America and some parts of Africa), and there are several predisposing factors, such as genetic background, poor oral hygiene and low socioeconomic status. ${ }^{2}$ The lesions are located mainly on the lower labial and buccal mucosa and, less frequently, the tongue. The differential diagnosis includes other conditions of the oral cavity, such as simple Fordyce spots, oral mucosal involvement of Crohn disease, mucosal neuromas in multiple endocrine neoplasia type $2 B$ and fibroepithelial papules in multiple hamartoma syndrome (Cowden disease), as well as other HPV-induced lesions, such as condylomata acuminata or verrucae vulgares. ${ }^{1,2}$

Treatment options for multifocal epithelial hyperplasia comprise surgical removal, laser excision, electrocautery, cryotherapy and topical agents such as imiquimod, retinoic acid or trichloroacetic acid. However, treatment is not always required, as most lesions are asymptomatic and frequently undergo spontaneous regression; there is no tendency to malignant transformation. ${ }^{2}$ We offered our patient treatment with laser therapy or trichloroacetic acid, but the parents declined therapy as he did not have clinical symptoms.

\section{References}

1. Said AK, Leao JC, Fedele S, Porter SR. Focal epithelial hyperplasia - an update. J Oral Pathol Med 2013;42:435-42.

2. de Castro LA, de Castro JG, da Cruz AD, et al. Focal epithelial hyperplasia (Heck's Disease) in a 57-year-old Brazilian patient: a case report and literature review. J Clin Med Res 2016;8:346-50.

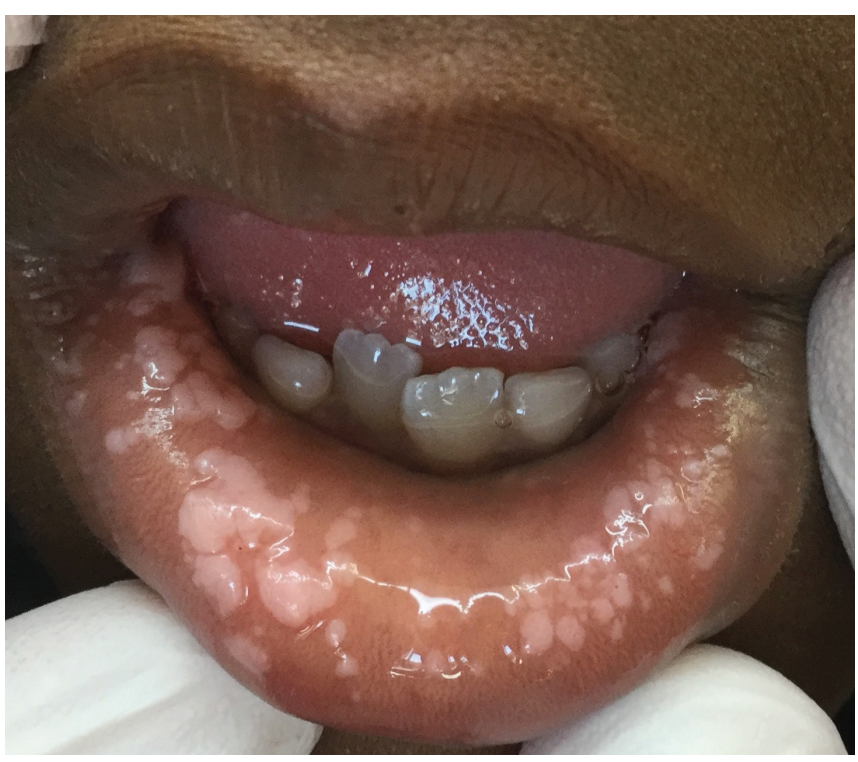

Figure 1: Multifocal epithelial neoplasia on the lower labial mucosa of a 7-year-old boy with Heck disease, manifesting as numerous flat, white papules and coalescing plaques.

\section{Competing interests: None declared.}

This article has been peer reviewed.

The authors have obtained consent from the patient's parents.

Affiliations: Department of Dermatology, Venereology, and Allergology (Kreuter), Helios St. Elisabeth, Hospital Oberhausen, University Witten/Herdecke, Witten, Germany; National Reference Center for Papilloma- and Polyomaviruses (Silling), Institute of Virology, University of Cologne, Cologne, Germany

Correspondence to: Alexander Kreuter, a.kreuter@derma.de

Clinical images are chosen because they are particularly intriguing, classic or dramatic. Submissions of clear, appropriately labelled highresolution images must be accompanied by a figure caption. A brief explanation (300 words maximum) of the educational importance of the images with minimal references is required. The patient's written consent for publication must be obtained before submission. 